\title{
Anti-seizure medication is not associated with an increased risk to develop cancer in epilepsy patients
}

\author{
Jenny Stritzelberger ${ }^{1} \mathbb{D}$. Johannes D. Lang ${ }^{1} \cdot$ Tamara M. Mueller $^{1} \cdot$ Caroline Reindl $^{1}$. Vivien Westermayer ${ }^{1}$. \\ Karel Kostev ${ }^{2}$ Hajo M. Hamer ${ }^{1}$
}

Received: 4 November 2020 / Revised: 18 December 2020 / Accepted: 19 December 2020 / Published online: 23 January 2021

(c) The Author(s) 2021

\begin{abstract}
Objective Whether anti-seizure medication (ASM) increases the risk for cancer has been debated for decades. While for some ASM, a carcinoma-promoting effect has been suspected, carcinoma-protective effects have been shown for other ASM. However, the issue remains unresolved as data from preclinical and clinical studies have been inconsistent and contradictory. Methods We collected anonymous patient data from practice neurologists throughout Germany between 2009 and 2018 using the IMS Disease Analyzer database (QuintilesIMS, Frankfurt, Germany). People with epilepsy (PWE) with an initial cancer diagnosis and antiepileptic therapy prior to the index date were 1:1 matched with a control group of PWE without cancer according to age, gender, index year, Charlson Comorbidity Index, and treating physician. For both groups, the risk to develop cancer under treatment with different ASMs was analyzed using three different models (ever use vs. never use (I), effect per one (II) and per five therapy years (III).

Results A total of 3152 PWE were included (each group, $n=1,576$; age $=67.3 \pm 14.0$ years). The risk to develop cancer was not significantly elevated for any ASM. Carbamazepine was associated with a decreased cancer risk (OR Model I: 0.699, $p<.0001$, OR Model II: 0.952, $p=.4878$, OR Model III: 0.758, $p<.0004$ ).
\end{abstract}

Significance Our findings suggest that ASM use does not increase the risk of cancer in epilepsy patients.

Keywords Epilepsy · Antiseizure medication · Carcinogenicity · Carbamazepine

\section{Abbreviations}

ASM Anti-seizure Medication

ATC Anatomical Therapeutic Chemical

CBZ Carbamazepin

CCI Charlson Comorbidity Index

GP General Practitioner

HDAC Histone deacetylase

LEV Levetiracetam

OR Odds Ratio

Supplementary Information The online version of this article (https://doi.org/10.1007/s00415-020-10379-4) contains supplementary material, which is available to authorized users.

Jenny Stritzelberger

jenny.stritzelberger@uk-erlangen.de

1 Department of Neurology, Epilepsy Center, FriedrichAlexander-Universität Erlangen-Nürnberg (FAU), Schwabachanlage 6, 91054 Erlangen, Germany

2 IQVIA, Epidemiology, Frankfurt am Main, Main Airport Center, Unterschweinstiege 2-14, 60549 Frankfurt am Main, Germany

$\begin{array}{ll}\text { PB } & \text { Phenobarbital } \\ \text { PHT } & \text { Phenytoin } \\ \text { PWE } & \text { People with Epilepsy } \\ \text { VPA } & \text { Valproate }\end{array}$

\section{Introduction}

The relationship between epilepsy and cancer has been the subject of considerable debate for a long time [1,2]. Besides epilepsy itself as a risk factor for cancer in persons with epilepsy (PWE) due to diagnostic procedures or the lifestyle of PWE, especially the propensity of anti-seizure medication (ASM) to promote or protect against cancer has been discussed in several previous animal and epidemiological studies with contradictory results [3-5].

Phenobarbital (PB), for example, has been under suspicion to promote liver cancer in rodents [6, 7], but so far, epidemiologic data did not support this relationship in humans [8]. Phenytoin (PHT) was classified as possibly carcinogenetic by the International Agency for Research on Cancer 
due to induction of lymphoma and liver cell cancer in rats $[9,10]$, and a few case studies reported association between PHT and certain cancer types in humans. A retrospective study assessing long-term treatment with benzodiazepines found a slightly increased cancer risk for clonazepam [11]. High doses of gabapentin were associated with pancreatic tumors in a rat model [12], but clinical data did not sufficiently support a potential carcinogenic effect of gabapentin in humans [13]. Therefore, clinical evidence for carcinogenicity in humans is neither consistent nor sufficient [5].

On the other hand, ASM like valproate (VPA) and carbamazepine (CBZ) has been reported to exert anti-proliferative effects on certain cancer cell lines via histone deacetylase (HDAC) inhibitory activities [14, 15]. PHT and CBZ inhibited Prostate-Specific-Antigen secretion in vitro [16]. Another study found protective effects against bladder cancer for PB therapy in smokers [17]. However, it remained again unclear whether these experimental results translate to clinically relevant cancer-suppressive effects of these drugs [5, 14, 18-21].

Taken together, despite a great body of preclinical data and mechanistically plausible considerations, evidence for human carcinogenicity is inconsistent and sparse and the association between ASM and cancer risk remains still unclear.

Our aim was, therefore, to evaluate the safety of longterm use of various ASM in terms of potential carcinogenetic effects. We retrospectively investigated the association between ASM prescriptions and the incidence of various cancer types in a large German cohort.

\section{Materials and methods}

\section{Database}

This study was based on data from the Disease Analyzer database (IQVIA $®$ ), which compiles drug prescriptions, diagnoses, and basic medical and demographic data obtained directly and in anonymous data format from computer systems used in practices of general practitioners and specialists throughout Germany [22]. The database covers approximately 3\% of all outpatient practices in Germany. Diagnoses (according to International Classification of Diseases, 10th revision [ICD-10]), prescriptions (according to Anatomical Therapeutic Chemical (ATC) Classification system), and the quality of reported data are monitored by IQVIA. In Germany, the sampling methods used to select physicians' practices are appropriate for obtaining a representative database of general and specialized practices [22].

\section{Study population}

This retrospective case-control study included patients with a diagnosis of epilepsy (ICD 10: G40) and a documented cancer diagnosis with the initial cancer diagnosis serving as the index date (ICD-10: C00-C97) from one of 1227 general practitioners between January 2009 and December 2018 (index date). Further inclusion criteria were as follows: (1) age 18-90 years at index date; (2) observation time of at least 12 months prior to the index date, and (3) epilepsy diagnosis and at least one ASM prescription prior to the index date (Fig. 1).

Using the same database, epilepsy patients without cancer were matched $(1: 1)$ to cancer cases by sex, age, index year, treating physician and a revised version of the Charlson Comorbidity Index (CCI) as a generic marker for comorbidity; the CCI describes 22 comorbid conditions where each condition is assigned a score from 1 to 6 depending on the risk of dying from it like congestive heart failure, diabetes, peripheral vascular disease or dementia [23]. The index date for the controls was a randomly selected visit date between 2009 and 2018 (Fig. 1).

\section{Study outcomes and covariates}

The main outcome of the study was the association between the ASM use and cancer diagnosis. The prescriptions of all drugs licensed and marketed for the treatment of epilepsy in Germany in the study period were included. This comprised all drugs listed under the ATC-code N03 "Antiepileptics" when they were available in Germany during the study period. Thus, the analyses included carbamazepine, clonazepam, gabapentin, lacosamide, lamotrigine, levetiracetam, oxcarbazepine, phenobarbital, phenytoin, pregabalin, primidone, topiramate, and valproate.

\section{Statistical analyses}

Descriptive analyses were performed for demographic and clinical variables, and the differences between cases and controls were evaluated using chi-squared tests for categorical variables and the Wilcoxon signed-rank test for age and CCI. Three regression models were used to analyze the association between ASM use and cancer risk. ASMs were included as a dichotomous variable in Model 1 (ever versus never use), as a continuous variable in Model 2 (therapy duration in years), and as dichotomous variable in Model 3 (at least 5 years of therapy vs. $<5$ years of therapy). Regression models were performed separately for cancer in total as well as most frequent cancer sites including digestive organs, respiratory organs, skin, breast and prostate. In the regression analyses, odds ratios $(\mathrm{OR})>1.0$ indicated a 
Fig. 1 Flow chart of the study cohort, showing inclusion criteria and numbers of included epilepsy patients in both groups. $\mathrm{ASM}=$ antiseizure medication

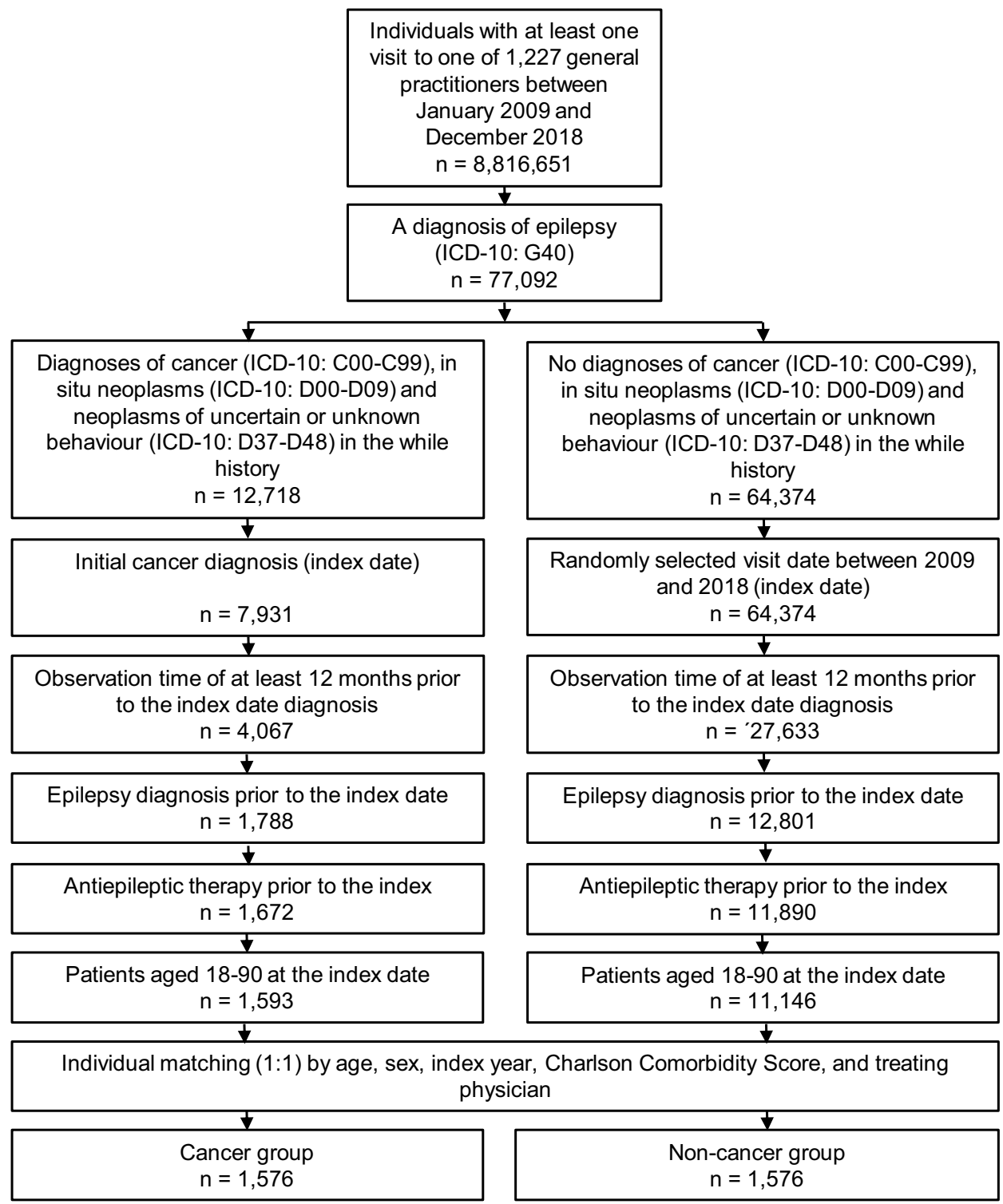

cancer-promoting effect while an $\mathrm{OR}<1.0$ implied a protective effect of the ASM observed. Results were regarded relevant when the significance level was $<0.01$ and $\mathrm{OR}$ were $<0.85$ or $>1.15$, respectively. A p value of $<0.05$ was considered statistically significant. All analyses were carried out using SAS 9.4 (SAS Institute, Cary, USA).

\section{Results}

\section{Patient characteristics}

From a total of 77,092 PWE in our database, 1576 PWE fulfilled the inclusion criteria (Fig. 1). The mean age was 67.3 years, with $70.1 \%$ being older than 60 years. $44.5 \%$ were females. The CCI was 3.7. 1576 PWE served as matched controls. The average duration of administration of ASM prior to the cancer diagnosis was 9.4 years (SD 5.8) with a maximal duration of 27.2 years.

\section{Anti-seizure medication}

Levetiracetam (LEV) was the most frequently administered ASM in both groups (cancer group: $36.0 \%$, non-cancer group: $32.2 \%$; $p<0.027$ ), followed by CBZ (24.6 vs. $30.9 \%$; $p<0.001)$, VPA (21.5 vs. $21.0 \% ; p<0.760)$ and LTG $(14.3$ vs. $15.1 \% ; p<0.546)$. There were no significant differences regarding the administration of specific ASM in both groups except for CBZ which was prescribed more frequently in the non-cancer group (Table 1). 
Table 1 Proportions of different antiseizure drugs prescribed in epilepsy patients (cancer patients and non-cancer controls)

\begin{tabular}{lllr}
\hline Variable & $\begin{array}{l}\text { Proportions in } \\
\text { cancer patients } \\
(\%)\end{array}$ & $\begin{array}{l}\text { Proportions in non- } \\
\text { cancer patients (\%) }\end{array}$ & P value \\
\hline $\mathrm{N}$ & 1573 & 1573 & \\
Carbamazepine & 24.6 & 30.9 & $<0.001$ \\
Clonazepam & 3.6 & 4.0 & 0.577 \\
Gabapentin & 10.9 & 11.2 & 0.820 \\
Lacosamide & 2.0 & 2.4 & 0.468 \\
Lamotrigine & 14.3 & 15.1 & 0.546 \\
Levetiracetam & 36.0 & 32.2 & 0.027 \\
Oxcarbazepine & 4.3 & 4.9 & 0.394 \\
Phenobarbital & 1.3 & 1.6 & 0.552 \\
Phenytoin & 6.2 & 8.2 & 0.033 \\
Pregabalin & 7.7 & 7.7 & 0.947 \\
Primidone & 4.8 & 4.5 & 0.673 \\
Topiramate & 2.7 & 3.3 & 0.348 \\
Valproate & 21.5 & 21.0 & 0.760 \\
\hline
\end{tabular}

\section{Risk of anti-seizure medication for cancer}

Exposure to ASM did not lead to a significant increase in total cancer incidence or in the organ-specific cancer subgroups in any of the three models (ever use vs never use, effect per 1 year of therapy, effect per 5 therapy years) with all OR being smaller than 1.1 (Table 2). The cancer risk did not differ in patients taking a combination of two or more ASM compared to those on monotherapy (OR 0.74, CI $0.49-1.11, p<0.147$ ).

Of note, CBZ was associated with a significant decreased incidence of cancer in Model I (ever used versus never use; OR 0.699, 95\% CI 0.586-0.833, $p<0.0001)$ and Model III (effect per 5 years of therapy, OR $0.758,95 \%$ CI $0.650-0.884, p<0.0004)$. In the cancer subgroups, this effect was found most pronounced for digestive organs and skin (Table S1).

\section{Discussion}

In this cohort study based on a German wide register from general practitioners, we looked at the possible associations of ASM use with cancer risk among PWE. The analyses followed the definition according to Shelby et al. that a relevant drug-cancer association should be significant, persistent over time, and carry a large relative risk [24]. Our main finding was that ASM administration did not significantly increase cancer incidence. This goes along with the results of several recently published epidemiological studies regarding this subject $[4,8,13,15,25,26]$.

Comorbidity in epilepsy patients is widespread. If cancer incidence is truly increased in PWE, various aspects of the epilepsies, such as diagnostic procedures or lifestyle factors, could be causal beside ASM use [3, 4]. To rule out confounding bias by epilepsy itself, people with epilepsy and ASM intake served as controls in the present study. In addition, we matched the groups according to age, sex, treating physician, index year and CCI to avoid further confounding patient or treatment characteristics. The $\mathrm{CCI}$ is a valid tool to measure the burden of prognostic comorbidities and is commonly used for risk adjustment in data analysis used in this study [23, 27]. The index does not explicitly include behavioral factors like smoking and alcohol use, eating habits or

Table 2 Association between antiseizure medication and the incidence of cancer in epilepsy patients in German general practices

\begin{tabular}{|c|c|c|c|c|c|c|c|c|c|c|c|c|}
\hline \multirow{3}{*}{$\begin{array}{l}\text { ASM } \\
\text { Carbamazepine }\end{array}$} & \multicolumn{4}{|c|}{ Model I: ever use versus never use } & \multicolumn{4}{|c|}{ Model II: Effect per year of therapy } & \multicolumn{4}{|c|}{ Model III: Effect per 5 therapy years } \\
\hline & \multirow{2}{*}{$\frac{\mathrm{OR}}{0.699}$} & \multicolumn{2}{|l|}{$95 \% \mathrm{CI}$} & \multirow{2}{*}{$\frac{p \text { value }}{<.0001}$} & \multirow{2}{*}{$\frac{\mathrm{OR}}{0.952}$} & \multicolumn{2}{|c|}{$95 \% \mathrm{CI}$} & \multirow{2}{*}{$\frac{p \text { value }}{0.4878}$} & \multirow{2}{*}{$\frac{\mathrm{OR}}{0.758}$} & \multicolumn{2}{|c|}{$95 \% \mathrm{CI}$} & \multirow{2}{*}{$\frac{p \text { value }}{0.0004}$} \\
\hline & & 0.586 & 0.833 & & & 0.923 & 0.982 & & & 0.650 & 0.884 & \\
\hline Clonazepam & 0.903 & 0.624 & 1.308 & 0.5910 & 0.995 & 0.911 & 1.087 & 0.6012 & 0.924 & 0.658 & 1.297 & 0.6493 \\
\hline Gabapentin & 0.904 & 0.716 & 1.140 & 0.3929 & 0.962 & 0.899 & 1.030 & 0.9345 & 0.913 & 0.729 & 1.144 & 0.4314 \\
\hline Lacosamide & 0.743 & 0.456 & 1.211 & 0.2332 & 1.120 & 0.913 & 1.375 & 0.2374 & 0.803 & 0.504 & 1.280 & 0.3565 \\
\hline Lamotrigine & 881 & 0.717 & 1.082 & 0.2256 & 0.964 & 0.910 & 1.021 & 0.2720 & 0.866 & 0.710 & 1.057 & 0.1573 \\
\hline Levetiracetam & 1.033 & 0.874 & 1.220 & 0.7072 & 1.005 & 0.957 & 1.056 & 0.0207 & 1.063 & 0.907 & 1.246 & 0.4535 \\
\hline Oxcarbazepine & 0.807 & 0.574 & 1.134 & 0.2168 & 1.017 & 0.928 & 1.114 & 0.0813 & 0.833 & 0.605 & 1.147 & 0.2625 \\
\hline Phenobarbital & 0.833 & 0.455 & 1.523 & 0.5525 & 0.969 & 0.835 & 1.124 & 0.5023 & 0.835 & 0.463 & 1.504 & 0.5477 \\
\hline Phenytoin & 0.684 & 0.516 & 0.908 & 0.0086 & 0.988 & 0.943 & 1.034 & & 0.816 & 0.641 & 1.039 & 0.0992 \\
\hline Pregabalin & 0.979 & 0.749 & 1.278 & 0.8740 & 0.940 & 0.862 & 1.024 & 0.3630 & 0.955 & 0.739 & 1.235 & 0.7265 \\
\hline Primidone & 1.042 & 0.743 & 1.461 & 0.8125 & 0.949 & 0.889 & 1.014 & 0.2533 & 1025 & 0.759 & 1.384 & 0.8705 \\
\hline Topiramate & 0.810 & 0.535 & 1.226 & 0.3184 & 1.046 & 0.925 & 1.183 & 0.1650 & 0.868 & 0.582 & 1.296 & 0.4885 \\
\hline Valproate & 0.937 & 0.779 & 1.126 & 0.4865 & 0.993 & 0.958 & 1.030 & 0.1958 & 0.964 & 0.815 & 1.140 & 0.6689 \\
\hline
\end{tabular}

$A S M$ antiseizure medication, $O R$ odds ratio, $C I$ confidence interval 
exposure to chemical noxae. However, these factors are risk factors for the comorbidities considered in the CCI.

We examined three models for each group and drug, one for any use effects (Model I, ever vs. never use) and two for long-term effects (Model II + III, effect per 1 or 5 therapy years). Carcinogenicity may be the result of genotoxic or non-genotoxic effects. For genotoxic effects, there is no safe exposure threshold or dose exists, i.e. short-term exposure may be sufficient to induce cancer. In contrast, nongenotoxic carcinogens have a safe exposure threshold and exert their carcinogenetic properties mostly in long-term use [28]. In our study, no ASM administration showed a positive drug-cancer association, neither for short-term nor long-term use. We detected a significant decrease in cancer risk under CBZ-therapy, namely for cancers of the skin and digestive system. Preclinical studies offer various models that would potentially explain cancer-suppressive effects of CBZ. For example, CBZ might prevent DNA damage induced by ionizing radiation, thus leading to a lower cancer risk (Kim et al., 2012). Additionally, CBZ may exert histone deacetylase (HDAC)-inhibitory activities [29]. HDACs are involved in modulating chromatin structure and gene expression; inhibition may increase tumor cell killing via complex mechanisms [30]. Still, our results should be carefully interpreted because the current study represents a retrospective association study which cannot prove causal relationships due to the design of the study. Another problem when dealing with this kind of retrospective data is the so-called protopathic bias which refers to the phenomenon that a drug may be started due to the first symptoms of a disease before its firm diagnosis and therefore, erroneously be suspected to cause the disease [31]. This error leads ultimately to overestimation of carcinogenetic effects of a drug. To avoid this, a time lag between exposure and cancer diagnoses may be included. Protopathic bias can be minimized by assessing various models over longer periods (effect per 1 and per 5 treatment years) which was applied to the present study. Regarding CBZ, our results may have been biased by similar effects. If diagnostics prompted by a first seizure show results suspicious for cancer, the patient might be rather put on a drug with low risk for interactions with oncological treatment strategies like LEV rather than on CBZ which has a high potential for interactions with various chemotherapies [32]. In fact, in our study, PWE with cancer were less likely to receive CBZ than PWE without cancer and LEV was more often used in PWE with cancer (see Table 1). Our retrospective design does, therefore, not allow to assume causal implications between CBZ and cancer suppression but might be useful generating new hypotheses for further research.

\section{Limitations}

We only included patients who were treated by GPs, who care for PWE in Germany in addition to neurologists [33]. Nevertheless, we cannot safely assume that we covered the entire spectrum of patients. Since we relied on ICD-codes, we also cannot exclude under- or misdiagnoses of epilepsy by GP doctors although ICD coding by physicians has been shown to be reliable for identifying PWE [34]. We excluded neurologists because we could not assume that neurologists accurately code cancer diagnoses in addition to the epilepsy diagnosis. Moreover, cancer patients may have been treated exclusively by specialists, e.g. gynecologists in the case of breast cancer, leading to a lack of GP documentation regarding this type of cancer. Unfortunately, it was not possible to match patients according to the severity of their epilepsy syndrome because our data are based on retrospective analyses of registers which did not provide data on seizure load. We did not test for dose-dependent effects because it would have made the groups too small for reasonable statistical analyses. This is certainly a weakness of our study and warrants further research. We were not able to record and match the patient groups for tumor stadium according to the TNMclassification either. This may have disguised effects of ASM on tumor severity.

\section{Conclusion}

In this retrospective analysis, we did not find any evidence that any of the investigated ASM increases the incidence of cancer in epilepsy patients. The decreased cancer risk under treatment with $\mathrm{CBZ}$ is of note but requires further prospective studies because bias cannot be excluded.

Funding Open Access funding enabled and organized by Projekt DEAL. No funding was received for this study.

Availability of data and material The datasets generated during and/or analysed during the current study are available from the corresponding author on reasonable request.

\section{Compliance with ethical standards}

Conflicts of interest Lang JD served on the speakers' bureau of Eisai and UCB. Kostev K is an employee of IQVIA, a company that primary focuses on analyses for pharmaceutical companies and runs the database used in this analysis. This study however, was not part of any business project. Hamer HM has served on the scientific advisory board of Arvelle, Bial, Desitin, Eisai, facetoface, GW, Novartis, Sandoz and UCB Pharma. He served on the speakers' bureau of or received unrestricted grants from Amgen, Ad-Tech, Bial, Bracco, Desitin, Eisai, GW, Nihon Kohden, Novartis, Pfizer, and UCB Pharma. 
Open Access This article is licensed under a Creative Commons Attribution 4.0 International License, which permits use, sharing, adaptation, distribution and reproduction in any medium or format, as long as you give appropriate credit to the original author(s) and the source, provide a link to the Creative Commons licence, and indicate if changes were made. The images or other third party material in this article are included in the article's Creative Commons licence, unless indicated otherwise in a credit line to the material. If material is not included in the article's Creative Commons licence and your intended use is not permitted by statutory regulation or exceeds the permitted use, you will need to obtain permission directly from the copyright holder. To view a copy of this licence, visit http://creativecommons.org/licenses/by/4.0/.

\section{References}

1. Neligan A, Bell GS, Johnson AL, Goodridge DM, Shorvon SD, Sander JW (2011) The long-term risk of premature mortality in people with epilepsy. Brain : a journal of neurology 134(Pt 2):388-395. https://doi.org/10.1093/brain/awq378

2. Nevalainen O, Ansakorpi H, Simola M, Raitanen J, Isojärvi J, Artama M et al (2014) Epilepsy-related clinical characteristics and mortality: a systematic review and meta-analysis. Neurology 83(21):1968-1977. https://doi.org/10.1212/wnl.0000000000001005

3. Adelöw C, Ahlbom A, Feychting M, Johnsson F, Schwartzbaum J, Tomson T (2006) Epilepsy as a risk factor for cancer. J Neurol Neurosurg Psychiatry 77(6):784-786. https://doi.org/10.1136/ jnnp.2005.083931

4. Kaae J, Carstensen L, Wohlfahrt J, Melbye M, Allison BH (2014) Epilepsy, anti-epileptic medication use and risk of cancer. Int J Cancer 134(4):932-938. https://doi.org/10.1002/ijc.28396

5. Singh G, Driever PH, Sander JW (2005) Cancer risk in people with epilepsy: the role of antiepileptic drugs. Brain : a journal of neurology 128(Pt 1):7-17. https://doi.org/10.1093/brain/awh363

6. Peraino C, Fry RJ, Staffeldt E (1971) Reduction and enhancement by phenobarbital of hepatocarcinogenesis induced in the rat by 2-acetylaminofluorene. Cancer Res 31(10):1506-1512

7. Diwan BA, Henneman JR, Rice JM (1995) Further evidence for promoter-dependent development of hepatoblastoma in the mouse. Cancer Lett 89(1):29-35. https://doi.org/10.1016/0304-3835(95)90154 $-\mathrm{X}$

8. La Vecchia C, Negri E (2014) A review of epidemiological data on epilepsy, phenobarbital, and risk of liver cancer. European journal of cancer prevention : the official journal of the European Cancer Prevention Organisation (ECP) 23(1):1-7. https://doi.org/10.1097/ CEJ.0b013e32836014c8

9. Toxicology and Carcinogenesis Studies of 5,5-Diphenylhydantoin (CAS No. 57-41-0) (Phenytoin) in F344/N Rats and B6C3F1 Mice (Feed Studies). National Toxicology Program technical report series. 1993;404:1-303.

10. Diwan BA, Henneman JR, Nims RW (2001) Enhancement of $\mathrm{N}$-nitrosodiethylamine-initiated hepatocarcinogenesis by phentoin in male $\mathrm{F} 344 / \mathrm{NCr}$ rats at a dose causing maximal induction of CYP2B. Int J Toxicol 20(2):81-87. https://doi.org/10.1080/10915 810151115191

11. Iqbal U, Nguyen PA, Syed-Abdul S, Yang HC, Huang CW, Jian WS et al (2015) Is long-term use of benzodiazepine a risk for cancer? Medicine 94(6):e483. https://doi.org/10.1097/md.000000000000048 3

12. Sigler RE, Gough AW, de la Iglesia FA (1995) Pancreatic acinar cell neoplasia in male Wistar rats following 2 years of gabapentin exposure. Toxicology 98(1-3):73-82. https://doi.org/10.1016/0300483x(94)02966-x
13. Friedman GD, Achacoso N, Habel LA. Gabapentin and Cancer Risk: Updated Findings from Kaiser Permanente Northern California. The Permanente journal. 2019;23. doi:https://doi.org/10.7812/ tpp/18-040.

14. Gefroh-Grimes HA, Gidal BE (2016) Antiepileptic drugs in patients with malignant brain tumor: beyond seizures and pharmacokinetics. Acta Neurol Scand 133(1):4-16. https://doi.org/10.1111/ane.12437

15. Salminen JK, Tammela TL, Auvinen A, Murtola TJ (2016) Antiepileptic drugs with histone deacetylase inhibition activity and prostate cancer risk: a population-based case-control study. Cancer Causes Control 27(5):637-645. https://doi.org/10.1007/s10552-016-0737-2

16. Abdul M, Hoosein N (2001) Inhibition by anticonvulsants of prostate-specific antigen and interleukin- 6 secretion by human prostate cancer cells. Anticancer Res 21(3b):2045-2048

17. Olsen JH, Wallin H, Boice JD Jr, Rask K, Schulgen G, Fraumeni JF Jr (1993) Phenobarbital, drug metabolism, and human cancer. Cancer Epidemiol Biomarkers Prev 2(5):449-452

18. Blaheta RA, Cinatl J Jr (2002) Anti-tumor mechanisms of valproate: a novel role for an old drug. Med Res Rev 22(5):492-511. https:// doi.org/10.1002/med.10017

19. Happold C, Gorlia T, Chinot O, Gilbert MR, Nabors LB, Wick W et al (2016) Does Valproic Acid or Levetiracetam Improve Survival in Glioblastoma? A Pooled Analysis of Prospective Clinical Trials in Newly Diagnosed Glioblastoma. Journal of clinical oncology : official journal of the American Society of Clinical Oncology 34(7):731-739. https://doi.org/10.1200/jco.2015.63.6563

20. Duenas-Gonzalez A, Candelaria M, Perez-Plascencia C, Perez-Cardenas E, de la Cruz-Hernandez E, Herrera LA (2008) Valproic acid as epigenetic cancer drug: preclinical, clinical and transcriptional effects on solid tumors. Cancer Treat Rev 34(3):206-222. https:// doi.org/10.1016/j.ctrv.2007.11.003

21. Kamarudin MNA, Parhar I (2019) Emerging therapeutic potential of anti-psychotic drugs in the management of human glioma: A comprehensive review. Oncotarget 10(39):3952-3977. https://doi. org/10.18632/oncotarget.26994

22. Rathmann W, Scheerer M, Rohwedder K, Busch S, Kostev K (2018) Changes in patient characteristics, glucose lowering treatment, glycemic control and complications in type 2 diabetes in general practices (Disease Analyzer, Germany: 2008-2016). Postgrad Med 130(2):244-250. https://doi.org/10.1080/00325481.2018.1421842

23. Quan H, Li B, Couris CM, Fushimi K, Graham P, Hider P et al (2011) Updating and validating the Charlson comorbidity index and score for risk adjustment in hospital discharge abstracts using data from 6 countries. Am J Epidemiol 173(6):676-682. https://doi. org/10.1093/aje/kwq433

24. Selby JV, Friedman GD, Fireman BH (1989) Screening prescription drugs for possible carcinogenicity: eleven to fifteen years of followup. Cancer Res 49(20):5736-5747

25. Takada M, Fujimoto M, Motomura H, Hosomi K (2016) Inverse Association between Sodium Channel-Blocking Antiepileptic Drug Use and Cancer: Data Mining of Spontaneous Reporting and Claims Databases. International journal of medical sciences 13(1):48-59. https://doi.org/10.7150/ijms.13834

26. Stettner M, Krämer G, Strauss A, Kvitkina T, Ohle S, Kieseier BC et al (2012) Long-term antiepileptic treatment with histone deacetylase inhibitors may reduce the risk of prostate cancer. European journal of cancer prevention : the official journal of the European Cancer Prevention Organisation (ECP) 21(1):55-64. https://doi. org/10.1097/CEJ.0b013e32834a7e6f

27. Schneeweiss S, Maclure M (2000) Use of comorbidity scores for control of confounding in studies using administrative databases. Int J Epidemiol 29(5):891-898. https://doi.org/10.1093/ije/29.5.891

28. Nohmi T. Thresholds of Genotoxic and Non-Genotoxic Carcinogens. Toxicol Res. 2018;34. 
29. Beutler AS, Li S, Nicol R, Walsh MJ (2005) Carbamazepine is an inhibitor of histone deacetylases. Life Sci 76(26):3107-3115. https ://doi.org/10.1016/j.1fs.2005.01.003

30. Batty N, Malouf GG, Issa JP (2009) Histone deacetylase inhibitors as anti-neoplastic agents. Cancer Lett 280(2):192-200. https://doi. org/10.1016/j.canlet.2009.03.013

31. Horwitz RI, Feinstein AR (1980) The problem of "protopathic bias" in case-control studies. Am J Med 68(2):255-258. https://doi. org/10.1016/0002-9343(80)90363-0

32. Khansur EM, Shah AH, Lacy K, Kuchakulla M, Komotar RJ (2018) Novel Immunotherapeutics for the Treatment of Glioblastoma: The Last Decade of Research. Cureus 10(1):e2130. https://doi. org/10.7759/cureus. 2130

33. Hamer HM, Dodel R, Strzelczyk A, Balzer-Geldsetzer M, Reese JP, Schöffski O et al (2012) Prevalence, utilization, and costs of antiepileptic drugs for epilepsy in Germany-a nationwide population-based study in children and adults. J Neurol 259(11):23762384. https://doi.org/10.1007/s00415-012-6509-3

34. Jetté N, Reid AY, Quan H, Hill MD, Wiebe S (2010) How accurate is ICD coding for epilepsy? Epilepsia 51(1):62-69. https://doi.org/ 10.1111/j.1528-1167.2009.02201.x

Publisher's Note Springer Nature remains neutral with regard to jurisdictional claims in published maps and institutional affiliations. 